\title{
Influence of free surfaces on microstructure evolution of radiation damage in Fe from molecular dynamics and object kinetic Monte Carlo calculations
}

\author{
Maria J. Aliaga ${ }^{* 1}$ I. Dopico ${ }^{2}$, I. Martin-Bragado², Maria J. Caturla,1 \\ ${ }^{1}$ Dept. Física Aplicada, Facultad de Ciencias, Fase II, Universidad de Alicante, Alicante E-03690 \\ ${ }^{2}$ IMDEA Materials Institute, C/Eric Kandel 2, 28906 Getafe, Madrid, Spain
}

Received ZZZ, revised ZZZ, accepted ZZZ

Published online ZZZ (Dates will be provided by the publisher.)

Keywords Monte Carlo, molecular dynamics, fusion materials, radiation damage

* Corresponding author: e-mail mj.aliaga@ua.es, Phone: +34 96590 3400, Fax: +34 965909726

The influence of surfaces on the evolution of damage of irradiated $\mathrm{Fe}$ is studied using object kinetic Monte Carlo with input from molecular dynamics simulations and $a b$ initio calculations. Two effects are analysed: the influence of traps and the initial distribution of damage in the cascade. These simulations show that for a trap concentration of around 100appm, there are no significant differences between defect concentrations in bulk and thin films. However, the initial distribution of defects plays an important role not only on total defect concentration but also on defect type, for the model used in this study.

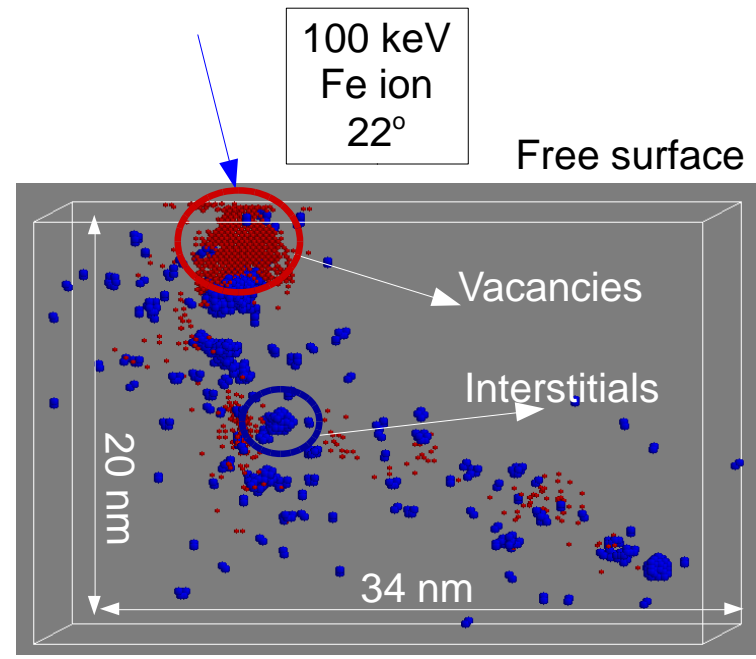

Damage produced by a $100 \mathrm{keV}$ Fe ion impinging a $\mathrm{Fe}$ thin film. Blue (dark) spheres are self-interstitials, red (light) spheres are vacancies. 


\section{Introduction}

Ion implantation is used to understand the effect of damage production in materials for applications in fusion and fission energy [1]. Unlike neutron irradiation, ion implantation allows for better control of variables such as irradiation temperature, dose, energy and, to some extent, dose rate. However, the extrapolation of results from ion implantation to neutron irradiation regarding defect production and microstructure evolution is not straightforward Effects such as differences in dose rate or sample thickness could affect significantly the evolution of the damage. The influence of surfaces on defect production, defect distribution and damage evolution needs to be understood in order to develop reliable models that can extrapolate the results from ion implantation to neutron irradiation conditions.

In this context, we present here a study, using object kinetic Monte Carlo (OKMC) calculations, of the effect of surfaces on defect evolution in irradiated Fe. We consider, on one hand, the interplay between trapping sites for defects and surfaces. And on the other hand the influence of the initial defect distribution of defects. It is well known from the early 1990s that damage produced close to the surface by an energetic ion in f.c.c. metals gives rise to defect structures that are significantly different from those produced in the bulk [2]. Recent simulations in Fe [3] and in Mo [4] have shown that this is also the case in b.c.c. metals, where large vacancy loops close to the surface have been identified. In order to study how this initial damage distribution (the picosecond damage distribution) affects the long term defect evolution we have used two databases of cascades, one obtained in bulk Fe and another one obtained in thin films with free surfaces [3]. OKMC calculations have been performed to calculate the defect concentration as a function of dose, as well as defect type and average cluster size, for different conditions: bulk cascades with periodic boundary conditions (which would correspond to the case of recoils within a bulk sample), bulk cascades in the presence of free surfaces (which would correspond to implantation at high energies such that the initial damage is not affected by the surfaces) and surface damage in a thin film (which would correspond to irradiation at low energy and damage close to surfaces). In section 2 of this paper we describe the model for damage accumulation and growth used in this work. Section 3 describes the results for the different conditions studied which are discussed in section 4 .

\section{Damage accumulation model}

Values obtained from ab initio calculations [5] and molecular dynamics simulations [6] are used for migration and binding energies of vacancies and self-interstitials, as shown in table 1 . All mobile defects in table 1 are consid- ered to move in three dimensions except for $1 / 2<111>$ loops which move in one-dimension.

All clusters can grow by addition of other defects of the same type. Recombination occurs between vacancy and interstitial type of defects, whether isolated or in clusters. In this particular model, the formation of $\langle 100\rangle$ loops occurs through the interaction between $1 / 2<111>$ loops, following the atomistic simulations of Marian et. al [7] and, more recently, Terentyev et al [8]. This model is not the only one possible for the formation of $\langle 100\rangle$ loops. Recently it was proposed that $<100\rangle$ loops can be formed from the nucleation of $\mathrm{C} 15$ clusters formed in the collision cascade $[9,10]$. However, the aim of this paper is not to discuss about the model for loop formation but about the effect of surfaces on a particular microstructure evolution model. For such a study we have selected the first model of loop formation; that of coalescence of $1 / 2<111>$ loops. In this model we consider that a $\langle 100\rangle$ loop can be formed as long as the two $1 / 2<111>$ loops interacting have similar sizes, with a maximum difference of $5 \%$. Once the $\langle 100\rangle$ loop is formed it can grow by adding new $1 / 2<111>$ loops, $<100>$ loops or small self-interstitial clusters.

Table 1 Migration energies $\left(E_{m}\right)$ and binding energies $\left(E_{b}\right)$ used for different defects in the OKMC model.

\begin{tabular}{lll}
\hline Defect type & $\mathrm{E}_{\mathrm{m}}(\mathrm{eV})$ & $\mathrm{E}_{\mathrm{b}}(\mathrm{eV})$ \\
\hline $\mathrm{V}$ & $0.67^{*}$ & \\
$\mathrm{~V} 2$ & $0.62^{*}$ & $0.3^{*}$ \\
$\mathrm{~V} 3$ & $0.35^{*}$ & $0.37^{*}$ \\
$\mathrm{~V} 4$ & $0.48^{*}$ & $0.62^{*}$ \\
$\mathrm{Vn}>4$ & Immobile & $* *$ \\
$\mathrm{I}$ & $0.34^{*}$ & \\
$\mathrm{I} 2$ & $0.42^{*}$ & $0.8^{*}$ \\
$\mathrm{I} 3$ & $0.43^{*}$ & $0.92^{*}$ \\
$\mathrm{I} 4$ & $0.3^{*}$ & $1.64^{*}$ \\
$\mathrm{In}>4{ }^{1 / 2<111>}$ & $0.06+0.11 / \mathrm{n}^{1.6}$ & $* *$ \\
$\mathrm{In}>4<100>$ & $\mathrm{Immobile}$ & $* *$ \\
\hline
\end{tabular}

$*[5] \quad * *[6]$

Self-interstitial clusters larger than 4 are considered to be $1 / 2<111>$ loops and therefore move with a very low migration energy barrier and in one-dimension. As a result, unless some traps are considered in the matrix, these loops quickly migrate to the surfaces and disappear leaving no residual damage. This, although a desirable situation from a radiation resistance point of view, it is not a realistic scenario. In the experiments there will always be some amount of impurities such as carbon. Therefore, it is necessary to include traps in the simulation. In this model $1 / 2<111>$ loops can be stopped when they find a trap, distributed randomly in the sample, with a binding energy of $1 \mathrm{eV}$. This trapped $1 / 2<111>$ loop is then immobile and can 
grow by addition of other loops or small self-interstitial clusters or monointerstitials.

The initial damage distribution produced by the energetic particle is obtained from molecular dynamics simulations [3]. We use two databases of cascade damage: one for damage produced by a recoil in the bulk, that is, simulations with periodic boundary conditions (PBC) and a second set of cascades produced by launching an energetic $\mathrm{Fe}$ ion on a Fe thin film (of about $5 \mathrm{~nm}$ ). All simulations were performed with the interatomic potential of Ackland and Mendelev [11].

The object kinetic Monte Carlo code MMonCa [13] was used in this study. Unlike other OKMC codes, where clusters are defined as an entity with a particular capture radius and number of defects but where the individual defect location is not considered, in MMonCa all positions of defects are followed. That is, a defect cluster does include all the individual positions of defects that form such a cluster. As a consequence, there is not a single capture radius associated to a cluster, but each defect has its own capture radius. In order to select the most appropriate value for this capture radius we have compared the defect morphology as obtained from the molecular dynamics simulations with that in the OKMC calculation, without allowing for any diffusion. Figure 1(a) shows the distribution of vacancies and self-interstitials obtained from a molecular dynamics simulations of a $50 \mathrm{keV} \mathrm{Fe} \mathrm{recoil} \mathrm{in} \mathrm{Fe.} \mathrm{Red} \mathrm{(light)} \mathrm{dots}$ correspond to the location of vacancies while blue (dark) dots correspond to self-interstitials. The location of these defects is calculated using a Wigner-Seitz cell algorithm. Figure 1(b) shows the location of those vacancies and selfinterstitials as given by the OKMC code when a capture radius of $0.4 \mathrm{~nm}$ is used. This capture radius is selected so that the number of monovacancies and monointerstitials matches those from the MD simulation (considering that two defects that are at first-nearest neighbours distance belong to the same cluster). If a larger capture radius is used in the OKMC, immediate recombination between vacancies and self-interstitials occurs and the total number of defects does not match the MD results. If a shorter capture radius is used, the number of defects identified as monovacancies or monointerstitials is larger than those used to identify clusters from the MD simulations. Nevertheless, one must keep in mind that this criterion for clustering of defects is somehow arbitrary, since other cut-off distances could be used. An evaluation of the damage distribution of defects from MD and OKMC, such as those shown in figure 1 , reveals that they are very similar, for this cut-off radius of $0.4 \mathrm{~nm}$.
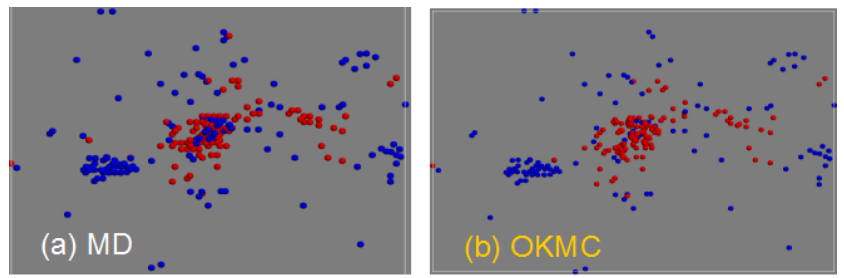

Figure 1 Defect distribution as obtained from MD simulations of a $50 \mathrm{keV} \mathrm{Fe}$ ion in $\mathrm{Fe}$ (a) and as initial conditions for the OKMC calculations (b). Red (light) spheres represent the location of vacancies, blue (dark) spheres are self-interstitials.

\section{Results}

Using the model described above, we have studied the evolution of the damage produced by energetic recoils in Fe. We have analysed, on one hand, the influence of surfaces on defect concentration and defect size, and on the other hand the influence of the initial defect distribution. As mentioned above, recent simulations of cascades in $\mathrm{Fe}$ have shown that damage produced by ion implantation with low energies $(\sim 100 \mathrm{keV})$ results in defect structures significantly different from those produced by the same energy recoils but in the bulk of the material [3]. One important difference is the formation of large $(>1 \mathrm{~nm})$ vacancy clusters of $\langle 100\rangle$ type when the damage is very close to the surface, together with smaller self-interstitial clusters as compared to bulk damage. Those results, however, only consider the first few picoseconds after the energy is transferred from the recoil to the lattice. Here, we follow the evolution of those defects produced in the cascade over longer times and under continuous irradiation with the use of the OKMC model.

Two data bases for cascade damage are used for these calculations both obtained with the same interactomic potential [11]: a database of $100 \mathrm{keV}$ recoils in bulk $\mathrm{Fe}$ and one of $100 \mathrm{keV} \mathrm{Fe}$ ion implantation in Fe thin films [3]. In order to decouple the effect of surfaces from the effect of the initial damage distribution we have performed three types of calculations: (1) bulk cascades with periodic boundary conditions, (2) bulk cascades in a thin-film (3) surface cascades in a thin-film. Thin films have a thickness of $50 \mathrm{~nm}$, similar to those used in in situ TEM studies. The simulation box is $200 \mathrm{~nm} \times 200 \mathrm{~nm} \times 50 \mathrm{~nm}$ and cascades are located randomly within this box with a dose rate of 8 $\mathrm{x} 10^{14}$ ions $\mathrm{m}^{-2} \mathrm{~s}^{-1}$. The same calculation is performed for two concentrations of traps in the lattice: 1 appm and 118 appm. Self-interstitial defects bind to these traps with a binding energy of $1 \mathrm{eV}$, which would mimic the effect of having carbon in the sample [13].

Figure 2 shows the total concentration of visible clusters as a function of dose for the case of 1 appm traps and the three different simulations: bulk cascades with PBC, bulk cascades in thin films and surface cascades in thin 
films. Figure 1(a) represents the concentration of $1 / 2<111\rangle$ loops while figure 1 (b) is the total number of $\langle 100\rangle$ loops. Clusters are considered visible if they have more than 100 defects ( $1 \mathrm{~nm}$ radius). As expected, the total number of clusters when PBC are used is higher than when we consider a thin film. Also, visible clusters appear at lower doses when PBC are used than in a thin film. This is due to the fast migration of $1 / 2<111>$ loops to surfaces in the thin film, that lower the total defect concentration. Note that we did not include any sinks in the simulations with PBC. Comparing the concentration of $1 / 2<111>$ loops for the two cases with free surfaces, bulk cascades and surface cascades, no significant differences are observed, the total concentration is quite similar in these two examples. However, there is an important difference regarding the formation of $<100>$ loops. When bulk cascades are considered almost no $\langle 100\rangle$ loops are formed but, when surface cascades are used, the concentration of $\langle 100\rangle$ loops is comparable to that of $1 / 2<111>$ loops. This is surprising at first since the size of the self-interstitial clusters in surface cascades is smaller than in the case of bulk cascades. Considering that the model that we are using here for $\langle 100\rangle$ loop formation is the recombination of two $1 / 2<111>$ loops, the reason for this difference has to be the higher probability of two small self-interstitial loops finding each other before finding the surface or a trap in the case of surface cascades.
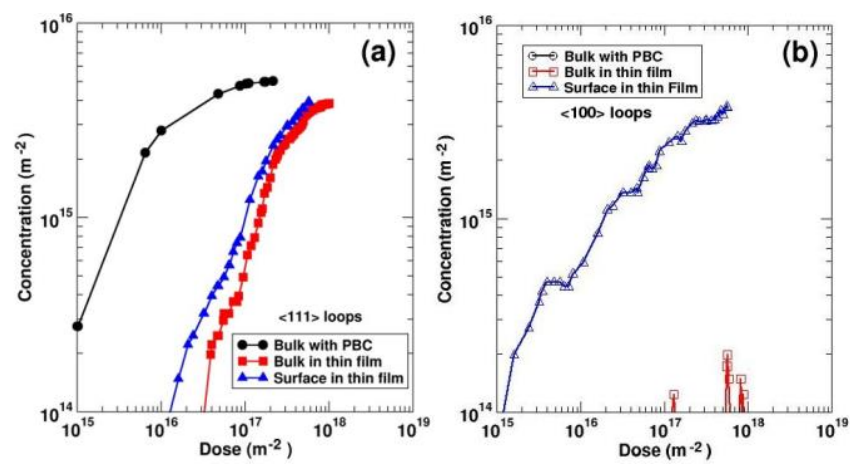

Figure 2 Concentration of visible (> 100 defects) self-interstitial clusters as a function of irradiation dose for 1 appm concentration of traps (a) $1 / 2<111>$ loops and (b) $<100>$ loops. Three different cases are considered: irradiation in bulk (squares), irradiation in a thin film with bulk cascades (circles) and irradiation in a thin film with surface cascades (triangles).

This, in fact, can be rationalized in terms of the distribution of defects within the cascade in the case of bulk damage or surface defects. Figure 3 shows one example of a cascade in the bulk (figure 3(a)) and a surface cascade (figure 3(b)). As it can be seen here, bulk cascades are spread over a longer range while surface cascades are more localized, in this particular case confined to a region of only 34 × $20 \times 26 \mathrm{~nm}$. Therefore, there is a much higher probability for two self-interstitial clusters to interact and form a $<100\rangle$ loop before reaching the surface or a trap in the case of surface cascades than in the case of bulk damage.
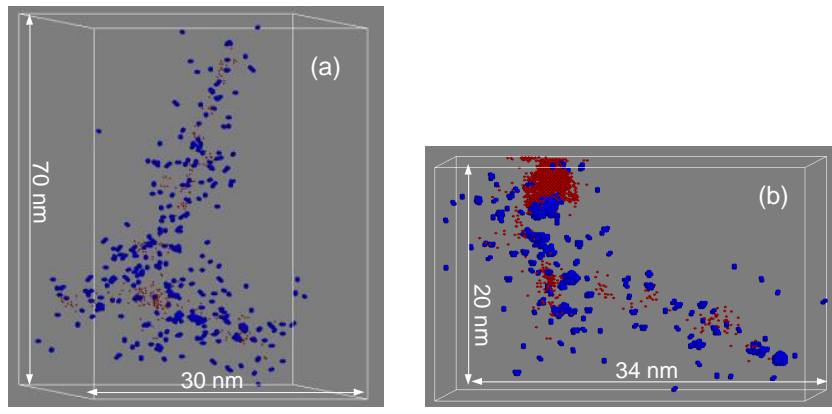

Figure 3 Defect distribution as obtained from MD simulations for $100 \mathrm{keV}$ and $22^{\circ}$ angle (a) Fe recoil in bulk Fe and (b) Fe ion implanted in Fe. Red (light) spheres represent the location of vacancies, blue (dark) spheres are self-interstitials.

We have also performed calculations for higher concentration of trapping sites, 118 appm. Figure 4 shows the concentration of $1 / 2<111>$ loops (figure 4(a)) and $\langle 100\rangle$ loops (figure 4(b)) for the three cases considered in this study. Now, the concentration of $1 / 2<111>$ loops is almost the same for all three cases. That is, the effect of the surface is negligible since all loops are trapped before they can reach the surface. However, the difference in the concentration of $\langle 100\rangle$ loops is still clear between bulk and surface cascades, since this is an effect of cascade damage distribution and therefore mostly independent of trapping concentration.
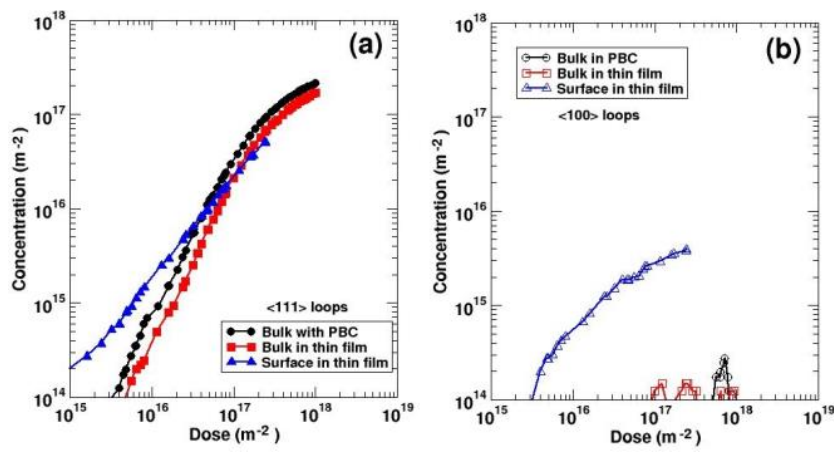

Figure 4 Concentration of visible (> 100 defects) self-interstitial clusters as a function of irradiation dose for 118 appm concentration of traps (a) $1 / 2<111>$ loops and (b) $<100>$ loops. Three different cases are considered: irradiation in bulk (squares), irradiation in a thin film with bulk cascades (circles) and irradiation in a thin film with surface cascades (triangles).

The differences in behaviour of $\langle 100\rangle$ loops with respect to $1 / 2<111>$ loops in this particular model are also observed in the average size of these loops as a function of dose, presented in figure 5. Results are shown for the cases 
of thin films with bulk cascades and with surface cascades. In the case of surface cascades, for any given dose, the average size of the clusters is larger than for the case of bulk cascades. Loops of the $1 / 2<111>$ type have a constant average size for low doses but after a certain dose, which is lower for surface cascades, the average size of these loops increases rapidly with dose. For the case of $<100\rangle$ loops, the average size remains constant for all doses studied here. The reason for these differences is the nucleation mechanism considered in this model. $\langle 100\rangle$ loops grow through the reaction between $1 / 2<111>$ loops of similar size, therefore, nucleation sites for these loops are constantly forming as long as the material is being irradiated. $1 / 2<111>$ loops are highly mobile, they can either migrate to the surface, interact to form $\langle 100\rangle$ loops or become trapped. When all trapping sites are saturated, no more nucleation sites for $1 / 2$ $<111>$ loops can be created and the ones that are already trapped can rapidly grow by the addition of new $1 / 2<111>$ loops. That gives rise to the rapid increase of the average size of these loops at high doses.

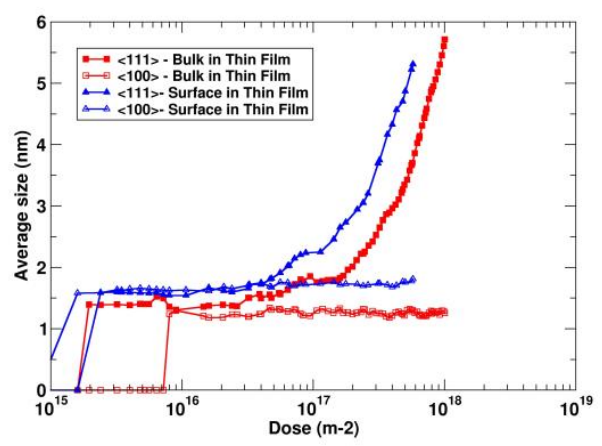

Figure 5 Average cluster size as a function of dose for the cases of bulk cascade in thin films and surface cascades in thin films. Values for $1 / 2<111>$ and $<100>$ loops.

4 Conclusions This work shows the importance of surfaces on the microstructure evolution of damage produced by irradiation. Surfaces act as sinks for defects, and, as expected, lower the total concentration of defects compared to bulk irradiation. This effect, however, will depend strongly on the purity of the sample and the presence of traps. More interestingly, if damage is produced very close to the surface, the distribution of this damage differs from that of bulk irradiation, resulting in, not only a different concentration of defects, but also differences in the type of damage that can be observed. In this particular model the differences are mostly related to the ratio of $1 / 2<111>$ to $<100>$ loops. Irradiation close to the surface favours the formation of $\langle 100\rangle$ loops due to the localization of the damage within the cascade.
Acknowledgements Simulations were carried out using the computer cluster of the Dept. of Applied Physics at the UA, the HPC-FF supercomputer of the Jülich Supercomputer Center (Germany) and the Helios supercomputer at Rokkasho (Japan). MJA thanks the UA for support through an institutional fellowship. The research leading to these results is partly funded by the European Atomic Energy Community's (Euratom) Seventh Framework Programme FP7/2007-2013 under grant agreement No. 604862 (MatISSE project) ) and in the framework of the EERA (European Energy Research Alliance) Joint Programme on Nuclear Materials. This work has been carried out within the framework of the EUROfusion Consortium and has received funding from the Euratom research and training programme 2014-2018 under grant agreement No 633053. The views and opinions expressed herein do not necessarily reflect those of the European Commission.

\section{References}

[1] J.L. Boutard, A. Alamo, R. Lindau, M. Rieth, C. R. Phys. 9 (2008) 287.

[2] M. Ghaly, R.S. Averback, Effect of viscous-flow on ion damage near solid-surfaces, Phys. Rev. Lett. 72 (1994) 364.

[3] M. J. Aliaga, R. Schäublin, J. F. Löffler, M. J. Caturla, Acta Materialia 101 (2015) 22-30.

[4] S. V. Starikov, Z. Insepov, J. Rest, A. Y. Kuksin, G. E. Norman, V. V. Stegailov, A. V. Yanikin, Radiation-induced damage and evolution of defects in Mo, Physical Review B 84, 104109 (2011). [5]C. C. Fu, J. Dalla Torre, F. Willaime, J.-L. Bouquet, A. Barbu, Nature Materials (2004)

[6] Soneda, N. \& Díaz de la Rubia, T. Phil. Mag. A 78, 995-1019 (1998)

[7] J. Marian and Brian D. Wirth PRL 88, 25 (2002).

[8] Haixuan Xu, Roger E. Stoller, Yuri N. Osetsky, and Dmitry Terentyev, PRL 110, 265503 (2013).

[9] M.-C. Marinica, F. Williaime, J.-P. Crocombette, PRL 108, 025501 (2012).

[10] Yongfeng Zhang, Xian-Ming Bai, Michael R. Tonks and S. Bulent Biner, Scripta Materialia 98 (2015) 5-8.

[11] G.J. Ackland, M.I. Mendelev, D.J. Srolovitz, S. Han, A.V. Barashev, Development of an interatomic potential for phosphorus impurities in alpha-iron, J. Phys.: Condens. Matter 16 (2004) S2629.

[12] I. Martin-Bragado, Antonio Rivera, Gonzalo Valles, Jose Luis Gomez-Selles, María J. Caturla Computer Physics Comunications (2013).

[13] V. Jansson, M. Chiapetto, L. Malerba, Journal of Nuclear Materials 442 (2013) 341-349. 